\title{
La ecografía y la entesis
}

\author{
Walter J. Spindler ${ }^{1}$, María Soledad Gálvez Elkin ${ }^{2}$, María Victoria Martire ${ }^{3}$
}

${ }^{1}$ Centro Médico Privado de Reumatología, Tucumán. ${ }^{2}$ Instituto de Cardiología Santiago del Estero. ${ }^{3}$ Reumatología y Enfermedades Autoinmunes Sistémicas, Hospital Italiano de La Plata.

En nombre del Grupo de Estudio de Ultrasonido de la Sociedad Argentina de Reumatología (GESAR Ultrasonido-ECOSAR)

Coordinación científica: Javier Rosa ${ }^{4}$, Santiago Ruta ${ }^{4}$

${ }^{4}$ Sección Reumatología, Servicio Clínica Médica, Hospital Italiano de Buenos Aires.

La ecografía aplicada al estudio de las enfermedades reumáticas es un área de constante crecimiento. La entesitis representa una de las principales características clínicas de las espondiloartritis. Con el advenimiento de nuevas terapias para el manejo de estas manifestaciones, es indispensable contar con herramientas que faciliten su estudio. La ecografía ha demostrado ser una técnica sensible y reproducible para la detección del compromiso inflamatorio a nivel de las entesis y existe evidencia acerca de su utilidad para el diagnóstico y monitoreo terapéutico de las espondiloartritis.

\section{Introducción}

La entesis representa la zona de inserción de tendones, ligamentos y cápsulas articulares en el hueso y corresponde tanto a la estructura insercional como al hueso mismo en el cual se inserta. Actualmente se denomina a estos tejidos "complejo órgano-entesal". Estas estructuras no sólo se afectan clínicamente en las espondiloartritis (EsA) sino que también representan fisiopatogénicamente el principal sitio "gatillo" para el desarrollo de estas patologías ${ }^{1}$.

Se denomina entesitis al compromiso inflamatorio de las entesis y es una de las principales manifestaciones clínicas dentro de todo el espectro de las EsA².

Debido a que la detección de entesitis mediante la evaluación clínica carece de precisión y sensibilidad, muchas veces son necesarios métodos de imágenes para reconocer el compromiso inflamatorio a nivel de los sitios de entesis en pacientes con EsA. La ecografía representa una de las técnicas más aceptadas debido a su bajo costo, rapidez de realización e inocuidad. Esta herramienta ha demostrado ser sensible y reproducible en la detección de entesitis ${ }^{3}$.

A continuación se describen aspectos actuales de la ecografía para la evaluación de las entesis en pacientes con EsA y sus principales utilidades.

\section{Técnica de evaluación por ecografía y lesiones elementales de las entesis}

Los sitios de entesis más comúnmente evaluados por ecografía en pacientes con EsA se localizan en los miembros inferiores, principalmente a nivel de la rodilla (cuádriceps distal y tendón rotuliano proximal y distal) y en el hueso calcáneo (tendón de Aquiles y fascia plantar). Otra localización frecuente para evaluar es el codo, ya sea en la inserción de los tendones extensores (epicóndilo), tendones flexores (epitróclea) y/o del tendón del tríceps (olécranon). En pacientes con Artritis Psoriásica (APs) es característico el compromiso de las entesis de los tendones extensores a nivel de las articulaciones interfalángicas distales ${ }^{4}$.

La evaluación de las entesis debe ser realizada utilizando abundante gel y preferentemente sin ejercer presión sobre el área a evaluar para evitar la compresión de la microvasculatura. La aplicación del Doppler de poder (DP) a nivel de las entesis se debe realizar con la región a estudiar en posición "neutra” y con la máxima relajación posible 5 .

Las posiciones y cortes ecográficos se resumen en la Tabla 1 (adaptada de Ruta S. y cols.) ${ }^{6}$.

El grupo de trabajo Outcome Measures in Rheumatology (OMERACT) ha definido a la entesopatía como una alteración hipoecogénica, generada por la pérdida de la arquitectura fibrilar normal y/o engrosamiento del ligamento o tendón en su inserción ósea, que puede contener focos hiperecoicos sugestivos de calcificaciones.

Las lesiones deben ser vistas en dos planos perpendiculares (transversal y longitudinal) y pueden mostrar o no señal DP o cambios óseos como entesofitos, erosiones y/o irregularidades ${ }^{7}$.

Existen otras lesiones elementales ecográficas, como las bursitis $^{8}$ y las alteraciones del fibrocartílago que no forman parte de la definición propuesta por OMERACT, pero son consideradas como parte de la patología inflamatoria a nivel de las entesis. 


\begin{tabular}{|c|c|c|c|}
\hline ZONA & ENTESIS EVALUADA & CORTE LONGITUDINAL Y TRANSVERSAL & POSICIÓN DEL PACIENTE \\
\hline \multirow[t]{3}{*}{ CODO } & $\begin{array}{l}\text { Tendones Extensores del carpo } \\
\text { a nivel del epicóndilo }\end{array}$ & A nivel lateral & \multirow{2}{*}{$\begin{array}{l}\text { Sentado con miembro superior sobre la } \\
\text { camilla con antebrazo flexionado sobre } \\
\text { el brazo a } 90^{\circ}\end{array}$} \\
\hline & Tendón del Tríceps a nivel del olécranon & A nivel posterior & \\
\hline & $\begin{array}{l}\text { Tendones de los Flexores del } \\
\text { carpo a nivel de la epitróclea }\end{array}$ & A nivel medial & $\begin{array}{l}\text { Sentado con miembro superior sobre la } \\
\text { camilla con antebrazo extendido }\end{array}$ \\
\hline CADERA & Tendones glúteos a nivel del trocánter mayor & A nivel lateral & $\begin{array}{l}\text { En decúbito lateral, miembro inferior a } \\
\text { explorar con la rodilla semiflexionada, } \\
\text { miembro inferior contralateral extendido }\end{array}$ \\
\hline \multirow[t]{2}{*}{ RODILLA } & $\begin{array}{l}\text { Tendón del cuádriceps } \\
\text { en el polo superior de la rótula }\end{array}$ & A nivel suprapatelar & \multirow{2}{*}{$\begin{array}{l}\text { Decúbito supino, con la rodilla en distintos } \\
\text { grados de flexión }\left(30^{\circ}, 45^{\circ}, 70^{\circ}\right) \text { para la } \\
\text { evaluación en escala de grises. } \\
\text { Luego evaluar DP en posición neutra }\end{array}$} \\
\hline & $\begin{array}{l}\text { Tendón rotuliano a nivel del polo inferior } \\
\text { de la rótula y en la tuberosidad tibial }\end{array}$ & A nivel infrapatelar & \\
\hline \multirow[t]{2}{*}{ TOBILLO } & $\begin{array}{l}\text { Tendón de Aquiles en el polo } \\
\text { superior del calcáneo }\end{array}$ & $\begin{array}{l}\text { A nivel del polo } \\
\text { superior del calcáneo }\end{array}$ & \multirow{2}{*}{$\begin{array}{l}\text { Decúbito prono, con el pie colgando de la } \\
\text { camilla en flexión de } 90^{\circ} \text { para la evaluación } \\
\text { en escala de grises. Luego evaluar DP en } \\
\text { posición neutra }\end{array}$} \\
\hline & $\begin{array}{l}\text { Fascia plantar a nivel del polo } \\
\text { inferior del calcáneo }\end{array}$ & $\begin{array}{l}\text { A nivel del polo inferior } \\
\text { del calcáneo }\end{array}$ & \\
\hline
\end{tabular}

Tabla 1. Sitios de evaluación de entesis y técnica de evaluación (Adaptación Ruta y cols.)

A continuación se detallan las lesiones elementales ecográficas más características a nivel de las entesis en pacientes con EsA (Figura):

Alteraciones del espesor: aumento cuantitativo del espesor normal de la entesis, medido a nivel del punto de máximo espesor más cercano al sitio de inserción ósea. Los sitios más evaluados y sus respectivos puntos de corte son los siguientes:

Aponeurosis o fascia plantar $>4 \mathrm{~mm}$

Tendón de Aquiles $>5,29 \mathrm{~mm}$

Tendón rotuliano proximal o distal $>4 \mathrm{~mm}$

Tendón del cuádriceps $>6,1 \mathrm{~mm}$

Tendón del tríceps $>4,3 \mathrm{~mm}$

Alteraciones de la estructura:

Pérdida del patrón fibrilar: ausencia de la visualización del patrón ecogénico típico de las inserciones tendinosas, con pérdida del paralelismo ecogénico-anecogénico de sus fibras en el eje longitudinal y con pérdida de la imagen heterogénea en el corte transversal.

Áreas hipo-anecoicas focales que producen pérdida de la típica estructura "fibrilar" normal de la entesis. dón.

Alteraciones de la forma: aspecto fusiforme del ten-

Alteraciones de la bursa: la bursitis se define como un aumento del diámetro de la bursa mayor a $2 \mathrm{~mm}$, que se evidencia por un área anecogénica o hipoanecogénica en el área anatómica de la misma y puede ser compresible con el transductor. Esta estructura puede presentar señal DP en su interior. A pesar de no estar incluida en las definiciones

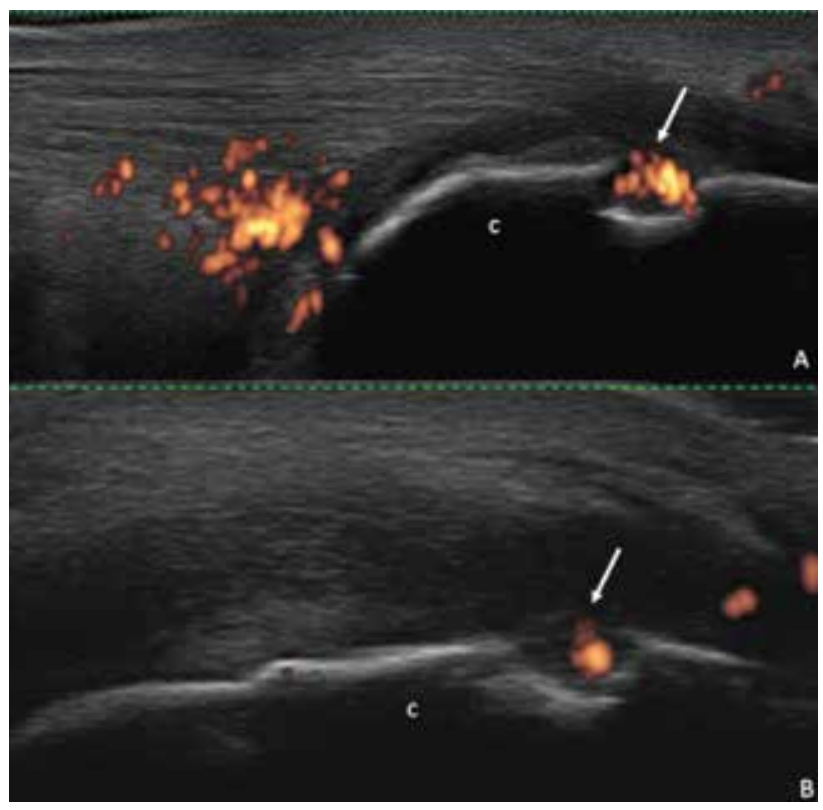

Figura. Inserción del Tendón de Aquiles en el polo superior del calcáneo (c). A. Corte longitudinal. Se observa alteración de la ecogenicidad del tendón con pérdida del patrón fibrilar normal a nivel insercional, erosión ósea (flecha) y aumento de la vascularización por la presencia de señal Doppler a nivel de la región de la bursa retrocalcánea y en la erosión ósea. B. Corte transversal. Se confirman la presencia de una erosión ósea a nivel del calcáneo. 
de OMERACT, las lesiones en la bursa están adquiriendo cada vez mayor importancia. Falcao y cols. demostraron que la presencia de la bursa retrocalcánea con un diámetro mayor a $2 \mathrm{~mm}$ presenta un bajo grado de sensibilidad $(19,8 \%)$ pero con una alta especificidad $(95,7 \%)$ para el diagnóstico de EsA. En este mismo estudio se observó que los controles sanos no presentaron señal DP, mientras que un $6,6 \%$ de los pacientes con EsA tuvieron este hallazgo a nivel de la bursa retrocalcánea?.

\section{Alteraciones óseas:}

Entesofitos: excrecencias óseas proyectadas hacia arriba, detectables a nivel de la zona distal de inserción tendinosa en el hueso, con o sin sombra acústica posterior. Las mismas se presentan como imágenes hiperecogénicas prominentes. Estas lesiones representan el hallazgo más frecuente en pacientes con Espondilitis Anquilosante $(\mathrm{EA})^{10}$.

Calcificaciones en el área de entesis: depósitos hiperecogénicos de distinto tamaño a nivel intratendinoso que puede generar o no sombra acústica posterior.

Erosiones óseas: interrupciones de la continuidad de la cortical ósea que provoca un defecto hacia abajo y se debe observar en dos planos (longitudinal y transversal).

Otras alteraciones óseas que pueden hallarse son las irregularidades del contorno óseo en la zona de entesis, con aspecto en "dientes de sierra" y la osificación de la entesis. Esta última se presenta como una imagen hiperecogénica con sombra acústica posterior.

Aumento de la vascularización: como consecuencia de la neovascularización y el aumento anormal de la vascularizacion debido al proceso inflamatorio, se puede visualizar la presencia de señal DP a nivel del área de entesis. La presencia de esta alteración ecográfica varía según la patología estudiada, siendo poco frecuente en la EA y con mayor prevalencia en pacientes con APs (6\% y 40,2\% respectivamente) $)^{10,11}$.

\section{Sistemas de puntaje ecográfico de las entesopatías en pacientes con espondiloartritis}

Existen diferentes sistemas de puntaje para la valoración de las entesis. Los mismos han sido diseñados para realizar una evaluación y estandarización más extensa del compromiso entesítico. El índice ecográfico de Balint y cols. ${ }^{12}$ denominado GUESS (Glasgow Ultrasound Enthesitis Scoring System) ha sido uno de los primeros sistemas de puntaje para la evaluacion de las entesis, y posteriormente se desarrollaron nuevos índices ${ }^{13-14}$. Actualmente, junto con el MASEI, ${ }^{8}$ son los más utilizados. En la Tabla 2 se analizan las diferencias entre los diferentes índices ecográficos.

Indice GUESS: el sistema de puntaje GUESS evalúa, en forma bilateral, cinco entesis de las extremidades inferiores: fascia plantar, inserción distal del tendón de Aquiles, tendón rotuliano en sus inserciones proximales y distales y cuádriceps distal (Tabla 2). Este índice contempla la presencia de cuatro lesiones elementales únicamente en escala de grises: grosor de la entesis, presencia de entesofitos, erosión y bursitis. Las mismas puntúan en forma dicotómica como 1 o 0 (presentes o ausentes), recordando que a nivel del polo inferior del calcáneo y del polo superior de la rótula no se evalúa la presencia de bursitis. El mayor puntaje posible a obtener es de 36 puntos, demostrando mayor afección a mayor puntaje obtenido ${ }^{12}$.

Índice MASEI (Madrid Sonographic Enthesis Index): este sistema de puntaje estudia las mismas entesis de las extremidades inferiores que el índice GUESS, y agrega la evaluación de la entesis distal del tendón del tríceps a nivel de su inserción en el olécranon. Además de las alteraciones en escala de grises, considera la presencia de DP. Las lesiones elementales del índice MASEI incluyen: grosor, calcificaciones, erosiones, bursitis y DP. Se determinó un valor de corte de 18 puntos para diferenciar entre pacientes sanos y pacientes con diagnóstico de EsA, alcanzando una sensibilidad de 83,33\%, especificidad de $82,76 \%$ y un likelibood ratio positivo (LR+) de $4,83^{8}$. Este sistema de puntaje resultó ser una herramienta útil para clasificar a los pacientes con APs, con una especificidad del $95 \%{ }^{15}$.

También mostró utilidad para el diagnóstico de EsA temprana ${ }^{16}$ y para evaluar el compromiso de entesis en pacientes con uveítis anterior recurrente ${ }^{17}$.

\section{Utilidad de la ecografía como herramienta diagnóstica en las entesopatías}

Una de las aplicaciones más importantes de la ecografía a nivel de las entesis es en el diagnóstico de las EsA. D'Agostino y cols. evaluaron la utilidad de los hallazgos ecográficos en las entesis de pacientes con sospecha diagnóstica de EsA. La presencia de al menos una entesis con señal de DP mostró una sensibilidad del 76,5\% y una especificidad del $81,3 \%$, con un $\mathrm{LR}+4,1$, para el diagnóstico final de EsA, considerando como estándar de oro al criterio del médico tratante ${ }^{18}$.

En pacientes con psoriasis cutánea, la aplicación de la ecografía de las entesis, utilizando el sistema de puntaje MASEI, con un valor de corte de 20 puntos, fue de utilidad para clasificar a aquellos pacientes con diagnóstico de $\mathrm{APs}^{15}$. Otro estudio evaluó el valor pronóstico de los hallazgos por ecografía para el desarrollo de AP en pacientes con psoriasis cutánea. El espesor del tendón cuadricipital y la mayor puntuación del GUESS basal fueron predictores para el desarrollo de APs ${ }^{19}$.

Marchesoni y cols. mostraron las principales características a nivel de las entesis en pacientes con fibromialgia y APs. En este último grupo, se encontraron mayores fenómenos inflamatorios (70\% vs. 23\%, respectivamente). La presencia de erosiones y señal DP fue muy específica de $\mathrm{APs}^{20}$. 


\begin{tabular}{|c|c|c|c|c|c|}
\hline ÍNDICE & ENTESIS EVALUADAS & \multicolumn{2}{|c|}{ LESIONES EN ESCALA DE GRISES (EG) } & DP & PUNTUACIÓN \\
\hline GUESS & $\begin{array}{c}\text { CUÁDRICEPS } \\
\text { DISTAL } \\
\text { ROTULIANO } \\
\text { PROXIMAL } \\
\text { ROTULIANO } \\
\text { DISTAL } \\
\text { AQUILES } \\
\text { FASCIA PLANTAR }\end{array}$ & \multicolumn{2}{|c|}{$\begin{array}{l}\text { GROSOR } \\
\text { ENTESOFITOS } \\
\text { EROSIONES } \\
\text { BURSITIS }\end{array}$} & NO & $\begin{array}{l}\text { PUNTUACIÓN MÍNIMA-MÁXIMA } \\
0-36\end{array}$ \\
\hline D'AGOSTINO & $\begin{array}{l}\text { ÍDEM GUESS + } \\
\text { TIBIAL ANTERIOR } \\
\text { EPICÓNDILO } \\
\text { MEDIAL } \\
\text { EPICÓNDILO } \\
\text { LATERAL }\end{array}$ & \multicolumn{2}{|c|}{ ÍDEM GUESS } & sí & $\begin{array}{c}\text { 1: DP EN LA UNIÓN CORTICAL } \\
\text { SIN LESIONES EN EG. } \\
\text { 2A: DP ASOCIADA A EDEMA Y/O DISMINUCIÓN } \\
\text { DE LA ECOGENICIDAD EN LA UNIÓN } \\
\text { CORTICAL EN EG. } \\
\text { 2B: HALLAZGOS EN EG DE ESTADIO } \\
\text { 2A SIN DP. } \\
\text { 3A: ESTADIO 2A + EROSIONES 0 } \\
\text { CALCIFICACIONES EN LA ENTESIS Y BURSITIS } \\
\text { (OPCIONAL) } \\
\text { 3B: HALLAZGOS EN EG DE ESTADIO 3A } \\
\text { SIN DP. }\end{array}$ \\
\hline $\begin{array}{l}\text { SPANISH } \\
\text { ENTHESITIS } \\
\text { INDEX (SEI) }\end{array}$ & ÍDEM GUESS & $\begin{array}{c}\text { AGUDAS } \\
\uparrow \text { GROSOR } \\
\downarrow \text { ECOGENICIDAD } \\
\text { EDEMA } \\
\text { BURSITIS }\end{array}$ & $\begin{array}{c}\text { CRÓNICAS } \\
\downarrow \text { GROSOR } \\
\text { CALCIFICACIONES } \\
\text { ROTURAS } \\
\text { EROSIONES ÓSEAS }\end{array}$ & NO & $\begin{array}{l}\text { LESIONES AGUDAS (0-36) } \\
+ \text { LESIONES CRÓNICAS }(0-40) \\
\text { PUNTUACIÓN MÍNIMA-MÁXIMA: 0-76 }\end{array}$ \\
\hline MASEI & GUESS + TRÍCEPS & \multicolumn{2}{|c|}{$\begin{array}{c}\text { GROSOR } \\
\text { ENTESOFITOS/CALCIFICACIONES } \\
\text { EROSIONES } \\
\text { BURSITIS } \\
\text { CAMBIOS ESTRUCTURALES }\end{array}$} & sí & $\begin{array}{l}\text { PUNTUACIÓN MÍNIMA-MÁXIMA: } \\
0-136\end{array}$ \\
\hline $\begin{array}{l}\text { BELGRADE } \\
\text { ULTRASOUN } \\
\text { D } \\
\text { ENTHESITIS } \\
\text { SCORE } \\
\text { (BUSES) }\end{array}$ & $\begin{array}{c}\text { GUESS } \\
+ \text { EPICÓNDILO } \\
\text { LATERAL }\end{array}$ & \multicolumn{2}{|c|}{$\begin{array}{c}\text { GROSOR } \\
\text { ENTESOFITOS } \\
\text { CAMBIOS ESTRUCTURALES } \\
\text { EROSIONES }\end{array}$} & sí & $\begin{array}{l}\text { PUNTUACIÓN MÍNIMA-MÁXIMA: } \\
\text { 0- } 132\end{array}$ \\
\hline
\end{tabular}

Tabla 2. Índices ecográficos para la evaluación de entesis en ESA.

\section{Detección de entesopatía subclínica}

La entesopatía subclínica implica la presencia de alteraciones ecográficas del órgano entésico, en ausencia de manifestaciones clínicas. La ecografía ha mostrado mayor sensibilidad que el examen físico para la evaluación del compromiso inflamatorio de las entesis. Balint y cols. demostraron la presencia de entesitis subclínica por ecografía en el $56 \%$ de los pacientes, comparada con un $22 \%$ mediante detección clínica, en pacientes con EsA ${ }^{12}$. Ruta y cols. mostraron que el $60,8 \%$ de pacientes asintomáticos con diagnóstico de EsA presentó al menos un signo de entesopatía evaluada por ecografía a nivel de las entesis de miembros inferiores ${ }^{21}$.
También se encontró compromiso subclínico de entesis en pacientes con enfermedad inflamatoria intestinal y uveítis anterior recurrente, con compromiso significativamente mayor de las entesis en comparación con controles $\operatorname{sanos}^{22,17}$.

En pacientes con diagnóstico de AP temprana ${ }^{23}$ y psoriasis cutánea se ha demostrado la presencia de entesitis subclínica. La presencia de estas lesiones estaría relacionada con el compromiso ungueal ${ }^{24}$.

De Miguel y cols. evaluaron la opinión de reumatólogos españoles sobre la utilidad de la ecografía en la toma de decisiones diagnósticas y terapéuticas en unidades reumatológicas integradas por miembros de la Sociedad Española de Reumatología. Ellos encontraron que tan 
sólo el 5,9\% de las ecografías eran dirigidas a la detección temprana de entesopatía. Sin embargo, el $85 \%$ de los encuestados refirió estar de acuerdo o muy de acuerdo sobre el uso de la ecografía para el diagnóstico y tratamiento de las entesitis en la práctica clínica diaria ${ }^{25}$.

\section{Monitoreo terapéutico por ecografía a nivel de las entesis}

La ecografía ha demostrado ser eficaz como guía para realizar procedimientos terapéuticos locales (intervencionismo de las entesitis) y ser una herramienta útil para evaluar los resultados de la terapéutica local y sistémica. Este monitoreo de las entesitis en pacientes con EsA es posible dada la posibilidad de aplicar sistemas de puntaje ecográficos y de cuantificar la actividad inflamatoria mediante el uso del DP.

Aydin y cols. demostraron que luego de dos meses de tratamiento con agentes biológicos anti-TNF $\alpha$ en pacientes con EA, se observó mejoría evaluada por ecografía en escala de grises y con DP, a nivel de la inserción en el calcáneo del tendón de Aquiles ${ }^{26}$. Naredo y cols., en un estudio que incluyó 197 pacientes con EsA, demostraron que los hallazgos ecográficos susceptibles al cambio luego de 6 meses de tratamiento con anti-TNF son: el aumento del espesor, los cambios en la estructura, la bursitis y el DP. No se encontraron cambios por ecografía en las lesiones consideradas clásicamente crónicas como las calcificaciones, erosiones óseas y entesofitos ${ }^{27}$.

Sin embargo, un estudio publicado posteriormente por De Miguel y cols. demostró que luego de 12 meses de seguimiento, se produjo una desaparición del $50 \%$ de las erosiones óseas en pacientes con EsA. Este estudio aplicó una técnica de ultrasonido bidimensional (2D) y tridimensional (3D) y podría ser una alternativa desafiante al concepto habitual de la erosión como una lesión crónica no modificable por el tratamiento ${ }^{28}$.

En un estudio publicado recientemente, Ruta y cols. evaluaron la respuesta terapéutica evaluada por ecografía en pacientes con EsA, por medio de un sistema de puntaje compuesto. El mismo incluyó la evaluación del compromiso articular, tendinoso y de entesis. Luego de tres meses desde el cambio o inicio del tratamiento, tanto el sistema de puntaje global como los componentes individuales del mismo, mostraron una reducción estadísticamente significativa y sensibilidad al cambio ${ }^{29}$.

\section{Consideraciones finales}

La ecografía permite evaluar de un modo eficaz el compromiso de las entesis en pacientes con EsA. Con el advenimiento de terapéuticas más eficaces para el tratamiento de la entesitis, es de gran importancia contar con herramientas que nos permitan una valoración objetiva de esta manifestación. Consideramos que para un adecuado uso de la información brindada por este método es necesario el conocimiento de las lesiones ecográficas elementales, el manejo de la técnica y cortes ecográficos recomendados, y la aplicación sistemática de los diferentes índices ecográficos descriptos para valoración de las entesitis. Utilizando adecuadamente estos preceptos es posible detectar entesitis en la mayoría de los pacientes con EsA y en aquellos pacientes con sospecha de esta entidad. Debemos recordar también que la ecografía tiene un gran potencial para el monitoreo terapéutico local y sistémico y como guía para la realización de procedimientos invasivos.

\section{Bibliografía}

1. Jacques P, Lambrecht $S$, Verheugen E, Pauwels E, Kollias G, Armaka M, et al. Proof of concept: enthesitis and new bone formation in spondyloarthritis are driven by mechanical strain and stromal cells. Ann Rheum Dis 2014; 73:437-45.

2. McGonagle D, Marzo-Ortega $\mathrm{H}, \mathrm{O}^{\prime}$ Connor P, Gibbon W, Hawkey P, Henshaw K, et al. Histological assessment of the early enthesitis lesion in spondyloarthropathy. Ann Rheum Dis. 2002; 61(6):534-7.

3. Alcalde M, Acebes JC, Cruz M, González-Hombrado L, Herrero-Beaumont G, Sánchez-Peranute O. A Sonographic Enthesitic Index of lower limbs is a valuable tool in the assessment of ankylosing spondylitis. Ann Rheum Dis. 2007; 66:1015-9.

4. Aydin SZ, Castillo-Gallego C, Ash ZR, Marzo-Ortega $\mathrm{H}$, Emery P, Wakefield RJ, et al. Ultrasonographic assessment of nail in psoriatic disease shows a link between onychopathy and distal interphalangeal joint extensor tendon enthesopathy. Dermatology. 2012; 225(3):231-5.

5. Gutierrez M, Filippucci E, Grassi W, Rosemffet M. Intratendinous power Doppler changes related to patient position in seronegative spondyloarthritis. J Rheumatol. 2010 May; 37(5):1057-9.

6. Ruta S, Mendonça JA, de la Cruz B LB, Cerón Villaquirán CE, Chiapas-Gasca K. La ecografía en las espondiloartropatías seronegativas: rol y avances. Rev. Chil. Reumatol 2012; 28(3):142-150.

7. Wakefield RJ, Balint PV, Szkudlarek M, Filippucci E, Backhaus M, D'Agostino MA, et al.; OMERACT 7 Special Interest Group. Musculoskeletal ultrasound including definitions for ultrasonographic pathology. J Rheumatol. 2005; 32(12):2485-7.

8. De Miguel E, Cobo T, Munoz-Fernandez S, NaredoE, Uson J, Acebes JC, et al. Validity of enthesis ultrasound assessment in spondyloarthropathy. Ann Rheum Dis. 2009; 68(2):169-74. 
9. Falcao S, de Miguel E, Castillo-Gallego C, Peiteado D, Branco J, Martín Mola E. Achilles enthesis ultrasound: the importance of the bursa in spondyloarthritis. Clin Exp Rheumatol. 2013; 31(3):422-7.

10. Spadaro A, Iagnocco A, Perrotta FM, Modesti M, Scarno A, Valesini G. Clinical and ultrasonography assessment of peripheral enthesitis in ankylosing spondylitis. Rheumatology (Oxford) 2011; 50: 2080-6.

11. Bandinelli F, Prignano F, Bonciani D, Bartoli F, Collaku L, Candelieri A. et al. Ultrasound detects occult entheseal involvement in early psoriatic arthritis independently of clinical features and psoriasis severity. Clin Exp Rheumatol. 2013; 31(2):219-24.

12. Balint PV, Kane D, Wilson H, McInnes IB, Sturrock $\mathrm{RD}$. Ultrasonography of entheseal insertions in the lower limb in spondyloarthropathy. Ann Rheum Dis. 2002; 61:905-10.

13. Milutinovic S, Radunovic G, Veljkovic K, Zlatanovic M, Zlatkovic Svenda M, Perovic Radak M, et al. Development of ultrasound enthesitis score to identify patients with enthesitis having spondyloarthritis: prospective, double-blinded, controlled study. Clin Exp Rheumatol. 2015 Sep 7.

14. D'Agostino MA, Said-Nahal R, Hacquard-Bouder C, Brasseur JL, Dougados M, Breban M. Assessment of peripheral enthesitis in the spondylarthropathies by ultrasonography combined with power Doppler: a cross-sectional study. Arthritis Rheum. 2003; 48(2):523-33.

15. Eder L, Jayakar J, Thavaneswaran A, Haddad A, Chandran V, Salonen D, et al. Is the MAdrid Sonographic Enthesitis Index useful for differentiating psoriatic arthritis from psoriasis alone and healthy controls? J Rheumatol. 2014; 41(3):466-72.

16. de Miguel E, Muñoz-Fernandez S, Castillo C, CoboIbanez T, Martin-Mola E. Diagnostic accuracy of enthesis ultrasound in the diagnosis of early spondyloarthritis. Ann Rheum Dis. 2011; 70(3):434-439.

17. Muñoz-Fernández S, de Miguel E, Cobo-Ibáñez T, Madero R, Ferreira A, Hidalgo MV, et al. Enthesis inflammation in recurrent acute anterior uveitis without spondylarthritis. Arthritis Rheum. 2009; 60(7):198590.

18. D'Agostino MA, Aegerter P, Bechara K, Salliot C, Judet O, Chimenti MS, Monnet, et al. How to diagnose spondyloarthritis early? Accuracy of peripheral enthesitis detection by power Doppler ultrasonography. Ann Rheum Dis. 2011; 70(8):1433-40.

19. Tinazzi I, McGonagle D, Biasi D, Confente S, Caimmi C, Girolomoni G, et al. Preliminary evidence that subclinical enthesopathy may predict psoriatic arthritis in patients with psoriasis. J Rheumatol. 2011;
38(12):2691-2.

20. Marchesoni A, De Lucia O, Rotunno L, De Marco G, Manara M. Entheseal power Doppler ultrasonography: a comparison of psoriatic arthritis and fibromyalgia. J Rheumatol Suppl. 2012; 89:29-31.

21. Ruta S, Gutierrez M, Pena C, Garcia M, Arturi A, Filippucci E, et al. Prevalence of subclinical enthesopathy in patients with spondyloarthropathy: an ultrasound study. J Clin Rheumatol 2011; 17(1):18-22.

22. Bandinelli F, Milla M, Genise S, Giovannini L, Bagnoli S, Candelieri A, et al. Ultrasound discloses entheseal involvement in inactive and low active inflammatory bowel disease without clinical signs and symptoms of spondyloarthropathy. Rheumatology (Oxford). 2011; 50(7):1275-9.

23. Bandinelli F, Prignano F, Bonciani D, Bartoli F, Collaku L, Candelieri A, et al. Ultrasound detects occult entheseal involvement in early psoriatic arthritis independently of clinical features and psoriasis severity.

24. Ash ZR1, Tinazzi I, Gallego CC, Kwok C, Wilson C, Goodfield M, et al. Psoriasis patients with nail disease have a greater magnitude of underlying systemic subclinical enthesopathy than those with normal nails. Ann Rheum Dis. 2012; 71(4):553-6.

25. de Miguel E, Andreu JL, Naredo E, Möller I; Grupo de Trabajo de Ecografía de la Sociedad Española de Reumatología (ECOSER). Ultrasound in rheumatology: where are we and where are we going? Reumatol Clin. 2014; 10(1):6-9.

26. Aydin SZ, Karadag O, Filippucci E, Atagunduz P, Akdogan A, Kalyoncu U, et al. Monitoring Achilles enthesitis in ankylosing spondylitis during TNFalpha antagonist therapy: an ultrasound study. Rheumatology (Oxford) 2010; 49(3):578-82.

27. Naredo E, Batlle-Gualda E, Garcia-Vivar ML, GarciaAparicio AM, Fernandez-Sueiro JL, Fernandez-Prada $\mathrm{M}$, et al. Power Doppler ultrasonography assessment of entheses in spondyloarthropathies: response to therapy of entheseal abnormalities. J Rheumatol 2010; 37(10):2110-7.

28. de Miguel E, Falcao S, Castillo C, Plasencia C, García M, Branco JC, et al. Enthesis erosion in spondyloarthritis is not a persistent structural lesion. Ann Rheum Dis. 2011; 70(11):2008-10.

29. Ruta S, Acosta Felquer ML, Rosa J, Navarta DA, García Monaco R, Soriano ER. Responsiveness to therapy change of a global ultrasound assessment in spondyloarthritis patients. Clin Rheumatol. 2015; 34(1):125-32. 